\title{
LA RESPONSABILIDAD SOCIAL DEL CONTADOR PÚBLICO EN EL DESEMPEÑO DE SU TRABAJO EN LA ACTIVIDAD MINERA
}

\author{
(Social responsibility of public accountant in carrying out his work in mining activity)
}

\section{*Ana Rocío Acevedo Pérez, **María Claudia Acevedo Pérez, ***Luis Alberto Rodríguez}

*Escuela de Contaduría Pública Uptc, **Grupo de investigación GICONT

(Recibido: el 27 de Febrero de 2013 y aceptado 22 de Octubre de 2013)

\section{Resumen:}

El profesional contable en los últimos tiempos, como actor principal, ha sido cuestionado en el desempeño de su actividad e incluso involucrado frecuentemente con escándalos financieros de grandes empresas, poniendo en tela de juicio la responsabilidad social de su profesión. Sin embargo, aquí el análisis de la profesión contable se dirige específicamente a mirar el comportamiento del contador en un sector especial como es el minero, principalmente en lo relacionado con la asesoría en la explotación del carbón.

En este artículo se presentan los resultados de la investigación realizada a los contadores públicos que prestan sus servicios de asesoría a empresarios del sector minero, se indaga sobre temas relacionados con el nivel y calidad de la asesoría que les proporcionan estos en los diferentes temas económicos, administrativos, fiscales, laborales, sociales y ambientales, en lo relativo al desarrollo de la actividad.

Del mismo modo, el contable tiene un compromiso ético, tanto con las organizaciones como con los diferentes grupos que se vinculan directa e indirectamente a estas, de mantener el equilibrio en las organizaciones mediante el suministro de información a las partes vinculadas sobre los derechos que le corresponden en el desarrollo de la actividad. Y como administrador de la información y gestor en la toma de decisiones contribuye a la generación del bienestar común.

Por último, la responsabilidad social del contador público va más allá del compromiso con el Estado y las organizaciones en las que labora, puesto que existe un compromiso con los demás grupos de interés, incluidos el medioambiente y la sociedad.

Palabras clave: responsabilidad social, contador público, grupos de interés, contabilidad social, fe pública, bienestar común.

\section{Abstract:}

The accounting professional in recent times, as a major player, has been questioned in the performance of his work and even frequently involved with corporate financial scandals, putting into question the social responsibility of his profession. However, here, the analysis of the accounting profession is directed specifically to watch the behavior of the accountant in a particular sector such as mining, mainly in relation to the advise in the exploitation of coal.

This paper presents the results of the investigation on public accountants who provide advisory services to entrepreneurs in the mining sector. It inquires also on issues related to the level and quality of the advice that they provide in different topics such as economic, administrative, tax, labor, social and environmental issues in relation to the development of the activity.

Similarly the accountant has an ethical commitment to both organizations and the different groups that are linked directly or indirectly to maintain balance in organizations through the provision of information to related parties on their rights that correspond to them in the development of the activity, and as an administrator and manager of information in decision-making contributes to the generation of the common welfare.

Finally, the social responsibility of the public accountant goes beyond the commitment to the State and the organizations in which they work, because there is a commitment to other stakeholders including the environment and society.

Keywords: social responsibility, public accountant, interest groups, social accounting, public trust, common welfare. 


\section{INTRODUCCIÓN}

La responsabilidad social del profesional contable ha sido un tema cuestionado en nuestros días, cada vez las exigencias se hacen más grandes debido al constante cambio de la normatividad y la necesidad de una inquebrantable actualización, además, el contador en cumplimiento de su función como profesional de una disciplina social, mantiene un compromiso no solo con las organizaciones sino también con los grupos de interés de la misma, por lo tanto su responsabilidad social se hace aun mayor.

El contador público debe velar por que exista equilibrio en las organizaciones y su entorno, ya que su asesoría es importante en aspectos organizacionales, administrativos, económicos, fiscales, laborales, sociales y ambientales. Una asesoría integral sobre estos aspectos permitiría generar más valor a su trabajo y mayores beneficios tanto para los empresarios como para las demás partes vinculadas directa e indirectamente a las organizaciones. Por esto, aquí se tratan inicialmente algunos aspectos teóricos que resaltan los cimientos de la profesión como ciencia social, como son la teoría del bien común, la ética del contador público, la contabilidad como ciencia social, la formación del contador y su influencia en la generación de una conciencia social, y algunos aspectos sobre responsabilidad social del profesional. También se exponen algunos de los resultados obtenidos mediante la investigación realizada, se enuncian algunas consideraciones finales, conclusiones y recomendaciones.

\section{METODOLOGÍA}

Soportando la investigación de la responsabilidad social del contador sobre una serie de teorías como el bien común, la ética del contador público, la contabilidad como ciencia social, entre otras, utilizamos como tipo de estudio el descriptivo y un método de investigación exploratorio, y como fuentes y técnicas para recolección de información, primarias y secundarias. Fuentes primarias como aplicación de cuestionarios, encuestas y entrevistas para identificar la responsabilidad social del contador en la actividad minera: y fuentes secundarias como revisión de bibliografía, exploración documental de textos y documentos referentes al tema de estudio, para proceder a su análisis e interpretación. La metodología utilizada fue adecuada para este tipo de investigación debido a que la responsabilidad social del profesional contable en este sector ha sido muy baja y se pretenden establecer antecedentes sobre este tema que ha sido poco investigado y que puede dar lugar a futuras investigaciones.

\subsection{Teoría del bien común}

Según Argadoña (1998, p. 4), el principio, el sujeto y el fin de la sociedad y de todas las instituciones debe ser la persona humana. Estamos ante una agrupación de personas que pueden conocer lo que la sociedad es, lo que les aporta y lo que necesita de ellos, en donde el bien común puede entenderse como el conjunto de supuestos o condiciones sociales que hacen posible a los miembros de la sociedad la realización de sus fines personales.
Dupré (1994), (citado por Rivera, 2011, p. 10-12) establece que el bien común es un bien que va más allá del bienestar individual.

Pérez (2000, p. 4) afirma que la teoría del bien común se apoya en el concepto clásico de bien: la empresa hace bien a muchas personas, a algunas, por obligación a otras, de modo más o menos involuntario. Esto en virtud de su obligación de contribuir al bien común, que va desde la empresa hasta el de la comunidad local, el país, toda la humanidad y también las generaciones futuras.

\section{2 Ética del contador publico}

Según Curvelo Hassán (2009, p. 86-88), se puede afirmar que lo asumido como ética en contaduría es un mero acto de entrenamiento a un individuo sobre lo que es bueno o es malo frente a la ley. También referencia a Foucault, de cara a la vivencia de la ética en esta profesión. A este respecto se precisa que la principal forma de aprehender la ética por parte del contador ha estado demarcada por los procesos de formación profesional y la enseñanza cultural. A su vez se tiene en cuenta la noción de Kant, que constituye el soporte teórico en Cortina para referirse a una ética que sea aplicable a las organizaciones. Ella nos invita a renunciar a la existencia de códigos o normas. Factor que ha predominado en el desarrollo de la profesión contable en Colombia, y aunque las leyes son indispensables, no resuelven los dilemas morales que trae consigo la generación de las riquezas a través del uso de los recursos naturales requeridos para el desarrollo de su objeto social.

La ética kantiana es una de las más importantes en la formación de la conducta ética y socialmente responsable del contador. Para ello es necesario que se fundamente en la buena intención, en la buena conciencia individual. La buena acción moral es la que constituye una buena intención de su acción profesional. La buena intención del contador equivale a decir que la toma de decisiones debe traslapar la lógica de la organización en la que prima la generación de riqueza.

La ética del profesional de la contaduría pública es un factor importante para determinar su manera de actuar en el desarrollo de su profesión, como lo manifiestan Ospina et al., (2011, p. 51). Efectivamente, según estos autores, para el profesional contable actuar éticamente no representa una tarea fácil, debido a que los intereses de los empresarios están centrados en incrementar la rentabilidad y el rendimiento económico. En muchos casos estos intereses presentan incompatibilidad con las prácticas socialmente responsables. Por lo tanto, el crear una conciencia de responsabilidad social en el contador es un tema que aunque ha sido muy cuestionado debido a los diferentes problemas en los cuales se le ha involucrado, aún es un asunto complejo de tratar, puesto que "El origen ético de la responsabilidad social implica un compromiso moral. Por tanto, se asume en un nivel personal, aunque tiene su origen en la conciencia colectiva." (Curvelo, 2008, p. 497) 


\subsection{La contabilidad como ciencia social}

Machado (2004, p. 272) define la contabilidad como un saber (conocimiento) que resulta de un hacer cognoscitivo (pensar) para resolver problemas y mejorar la calidad de vida humana; La contaduría es una práctica social (profesión) que lleva a cabo soluciones a problemas concretos y que se sustenta en una serie de campos de conocimiento.

La responsabilidad del contador público en una organización trasciende de revelar una situación financiera: "La responsabilidad ya no consiste únicamente en rendir cuentas sobre PCGA (principios de contabilidad generalmente aceptados) y de auditoría, sino que es necesario asumir las consecuencias sociales derivadas de las actuaciones profesionales" (Mantilla, 2005, p. 273). Se tienen unos compromisos y unos derechos frente a las organizaciones, como lo menciona Machado (2007, p. 82). El contador público es un agente de información, certificación y fiscalización, que debe garantizar la responsabilidad social informando acerca de su cumplimiento y vigilando su inclusión en las prácticas cotidianas de las organizaciones.

\subsection{La formación del contador público y responsabilidad social}

El desempeño profesional de un contador público depende en gran medida de las ideas que obtuvo durante el proceso de formación. Como lo afirmaba Carbal (2009, p. 81), la responsabilidad social del contador público está condicionada por las ideas impartidas desde los diferentes programas de contaduría pública del país. Hablar de una auténtica responsabilidad social en el contador público sería considerada una utopía, si se contempla el carácter de empresa que las mismas instituciones gubernamentales han querido darle a la universidad y a sus espacios de formación, en la que prevalecen los elementos económicos sobre los académicos. Donde "El ser social, que permitiría un ejercicio disciplinar y profesional con base en principios éticos, morales y de juicios fundamentales para la construcción de la confianza y dar fe pública, no es predominante en el proceso formativo." (Martínez, 2006, p. 11)

La responsabilidad social del contador público se debe crear e incentivar desde los espacios de formación. Como lo mencionan Esperanza et al. (2010, p. 1), en la actualidad el papel que juegan la universidad y la educación contable se limita a un proceso educativo que se asimila más a un adiestramiento para el trabajo meramente económico cuyas pautas son dictadas por el mercado laboral, más que a una formación integral para la vida y el desempeño dentro de una sociedad en base al ser, saber y saber hacer. Entonces la educación se está limitando a crear profesionales aptos para desempeñar una labor especifica, pero no en fomentar un compromiso para con la sociedad y la concienciación sobre la responsabilidad en el ejercicio profesional.
Como señala Franco Ruiz (2002, p. 49), el tradicional énfasis desarticulado y descontextualizado de conocimientos adquiridos por el contador público para desempeñarse en el campo de trabajo y sus habilidades para el hacer, resultan insuficientes a la complejidad del mundo y sus constantes cambios científico-tecnológicos.

Por esto, Salazar (2002, p. 3) hace referencia a que el contador público requiere entonces durante la formación profesional adquirir los elementos que le permitan conocer el manejo de los recursos, apoyar el proceso administrativo de los negocios, tomar decisiones financieras, evaluar la gestión, ejercer control sobre las operaciones, fundamentar las cargas tributarias, proveer la información que conforman las estadísticas y contribuir a la evaluación de beneficios e impacto social, que la actividad económica del ente en el cual se encuentra ejerciendo, representa para la comunidad.

Machado (2007, p. 85) afirma: “El Contador Público deberá ser el profesional liberal que garantice socialmente que la corporación ha sido capaz de responder a los diversos grupos a través de una gestión". Y con un sentido de compromiso social, agregamos.

\subsection{La responsabilidad social profesional del contador público}

El contador público, al igual que cualquier otro profesional, tiene un compromiso con la sociedad en el desarrollo de su profesión, la cual contribuye a la creación de condiciones más justas y adecuadas de acuerdo con la ley y orientadas al bienestar común.

El contador público, sin duda, tiene una responsabilidad social en el desarrollo de sus funciones, las cuales involucran una serie de compromisos, como lo menciona Pinzón (2012, p. 2-4).

Existen tres compromisos por parte del contador público, que se generan a raíz de sus funciones. Un compromiso social, un compromiso empresarial y un compromiso intelectual.

El compromiso social significa que ante la sociedad, el contable debe ser una persona íntegra, porque de su análisis se desprende la evaluación minuciosa de situaciones problemáticas, que inciden en las decisiones de los actores empresariales. El compromiso empresarial, en donde el contador público cumple con sus funciones dentro de la organización teniendo en cuenta que siempre debe primar el bienestar general sobre el bienestar particular y evaluando las consecuencias de la información financiera que genera y su interpretación. Y el compromiso intelectual, que hace referencia a la actualización permanente del profesional, debido a la normatividad cambiante, que de no estar actualizada podría llevar a grandes riesgos.
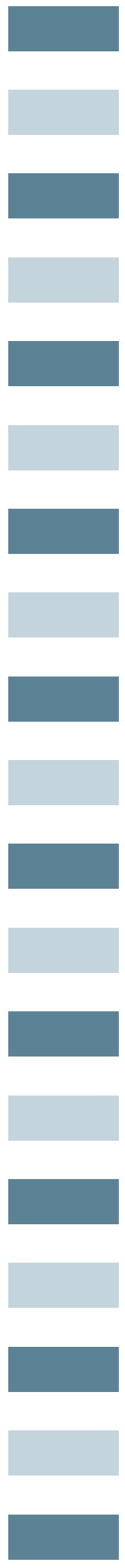


\subsection{Resultados}

En el desarrollo de la metodología propuesta se obtuvieron los resultados suficientes para afirmar que una vez aplicadas las encuestas a los empresarios mineros y contadores públicos asesores y entrevistas a los trabajadores y miembros de la comunidad, se encuentran divergencias entre las diferentes partes. Los empresarios manifiestan en su mayoría un nivel de asesoría bajo y en algunos casos nulo por parte del contador en los diferentes aspectos económicos, administrativos, fiscales, laborales, sociales y ambientales. Sin embargo se aplicaron las mismas preguntas en las diferentes posiciones y se encuentra que el profesional contable manifiesta lo contrario, presumiéndose que esto lo hacen como medida de defensa ante las preguntas planteadas. Es por esto que a continuación se realizan algunas comparaciones entre las diferentes respuestas que dieron contadores y empresarios:

Un número importante de empresarios mineros no cuentan con un contador público para su asesoría (37\% de los encuestados), ni utilizan sus servicios, ya que no los consideran necesarios; también afirman utilizar de una manera muy ocasional los servicios de un contador público, generalmente emplean sus servicios máximo cuatro veces al año para efectos de presentación de declaraciones y elaboración de balances.

A pesar de que la mayoría de los contadores encuestados manifiestan realizar visitas de por lo menos una vez al mes a la empresa minera (30\% de los contadores encuestados), hay una contradicción ya que un $51 \%$ de los empresarios mineros encuestados, manifiesta que nunca han hecho presencia en sus empresas y que, al contrario, son ellos quienes deben llevar su documentación a la oficina del contador en determinadas fechas en las que deben cumplir con una obligación tributaria.

Se encuentra que la mayoría de los contadores encuestados trabajan con mayor frecuencia en empresas comerciales (61 $\%)$, mientras que en otro tipo de organizaciones hay poca participación. Prestan sus servicios profesionales a las empresas mineras con poca frecuencia: los empresarios mineros afirman utilizar sus servicios: nunca un $35 \%$ y algunas veces $22 \%$, y los contadores públicos manifiestan que prestan sus servicios a los empresarios mineros: algunas veces $(39 \%)$, ante lo cual hay similitud entre las respuestas de las dos partes.

Los empresarios mineros encuestados que utilizan los servicios del contador público, manifiestan que no obtienen de su parte suficiente asesoría en cuanto a temas económicos, laborales, sociales y ambientales (un $29 \%$ manifiesta no recibir nada de asesoría en estos temas y un 23 $\%$ poca, a pesar de que los contadores encuestados sostienen asesorarlos medianamente en estos temas (30\%). Los empresarios mineros dicen que es mayor la asesoría que reciben sobre sus compromisos para con el Estado que para con los demás grupos de interés.
Se encuentra que el aspecto en el que el contador público brinda más apoyo a los empresarios mineros es en el tema fiscal, sobre cualquier otro aspecto, ya que lo afirman tanto los empresarios (en un $53 \%$ ) como los contadores encuestados (en el $65 \%$ ).

La mayoría de los contadores encuestados, conoce en gran parte el concepto de responsabilidad social (44\%) y considera que tienen una responsabilidad social profesional (39\% considera que totalmente, y un $26 \%$ en gran parte tienen responsabilidad social profesional); igualmente conoce en gran parte el concepto de grupos de interés ( $44 \%$ de los encuestados) y opina que como contadores públicos tienen una responsabilidad social para con los grupos de interés de la empresa que asesoran (44\% opinan que su responsabilidad para con los grupos de interés es mucha y un $17 \%$ consideran que es bastante).

También la mayoría de los contadores encuestados (39\%) piensa que en gran parte están contribuyendo al bienestar de los grupos de interés de las organizaciones en que trabajan a través de la asesoría que les brindan a los empresarios y que esta puede impactar bastante a los grupos de interés de la misma de una manera tanto positiva como negativa

Un $47 \%$ de los empresarios mineros, cuando tiene un problema relacionado con la productividad o rentabilidad de su actividad, prefiere utilizar los servicios de un profesional que esté más familiarizado con la actividad que ellos realizan. Es por esto que la mayoría prefieren utilizar la asesoría de un ingeniero de minas, y también se encuentra que es la única figura profesional que conocen los trabajadores mineros en las organizaciones en que trabajan, teniendo en cuenta las entrevistas que fueron aplicadas a los trabajadores de las empresas mineras.

Los contadores encuestados expresan que cuando intervienen en la gestión total de la información de la empresa minera, encuentran mayores problemas en lo relacionado con el tema laboral ( $22 \%$ de los encuestados), principalmente en cuanto a la determinación de bases equivocadas de cotización en seguridad social por diferencias en el salario devengado y también errores en la liquidación de prestaciones legales.

Los contadores encuestados declaran que entre sus otras funciones en la asesoría de las empresas mineras, brindan principalmente apoyo administrativo en la organización de la empresa minera que asesoran (35\%) y apoyo legal en cuanto a la solución de problemas $(26 \%)$,

Sin embargo, se encuentra que un $31 \%$ de los contadores encuestados considera que en ocasiones su trabajo se desvincula medianamente de las demás funciones de la organización, y un $30 \%$ opina que su trabajo se desvincula en gran parte.

También se halla que los contadores públicos no suministran información sobre los costos laborales de los empresarios mineros a quienes asesoran (56\% de los contadores encuestados), manifestación sobre la cual coincidieron con los empresarios mineros. 
Se encuentra que la asesoría a las empresas mineras es baja en temas económicos, sociales, ambientales y administrativos, pues el principal aspecto en que los contadores asesoran a estas organizaciones es en el tema fiscal; además existen falencias en el suministro de información a los empresarios mineros, ya que ellos no les dan a conocer en sus estados financieros las situaciones que no les generan beneficio para la actividad, ni les informan, en la mayoría de casos, sobre sus costos laborales, ni se han reunido nunca con los empresarios mineros para dar solución a temas laborales, sociales y ambientales de las organizaciones.

Un porcentaje importante de los contadores encuestados considera que no conoce bien la actividad que realiza la empresa minera que asesora, ni sus grupos de interés, lo cual está llevando a que su trabajo como asesor no se realice de la manera adecuada, ni genere la confianza necesaria a los usuarios de la información.

Muchos de los errores y omisiones sobre los que incurren los empresarios mineros, están relacionados con la falta de asesoría y conocimiento en determinadas situaciones, encontrándose que si existiera una mayor asesoría para las organizaciones tanto mineras como de cualquier otro sector, se mejorarían las condiciones de trabajo de las organizaciones y se garantizarían, tanto para los empresarios como para los demás grupos de interés, mayores beneficios.

El contador público, como profesional de una disciplina social, adquiere un compromiso profesional y personal con las organizaciones que labora, para que su trabajo se realice de una manera más integral en servicio del bien común. Es por esto que se resalta la importancia de que su asesoría sea un poco más amplia y que no se limite solo al aspecto fiscal, por lo tanto es importante que conozca bien la actividad sobre la cual asesora y los diferentes grupos de interés de la misma, informando sobre los compromisos que tienen las organizaciones para con cada uno, ya que su asesoría puede influir tanto positiva como negativamente sobre los mismos

\section{RECOMENDACIONES}

Es necesario que el profesional contable participe en la creación de responsabilidad social empresarial, desde el compromiso que adquiere con los empresarios de las organizaciones en que labora y con los demás grupos de interés de la organización, a favor del buen funcionamiento de cualquier organización y del bienestar común.

Es preciso que el contador amplíe su visión como profesional de una disciplina social, identificando la responsabilidad que tiene para con los diferentes grupos de interés de las organizaciones que asesora, y no limite su función dentro de las organizaciones al cumplimiento exclusivo de la ley.
Es necesario que el profesional dimensione su labor de asesor dentro de las organizaciones en los diferentes temas económicos, administrativos, fiscales, sociales y ambientales, más allá del cumplimiento de una labor específica.

Es importante que se resalte la responsabilidad social del contador público desde su proceso de formación, y que se tenga en cuenta tanto la parte técnica de la profesión como la importancia del análisis y la interpretación de la información.

Desde los centros educativos donde se forman los contadores públicos, se hace necesario que los temas de estudio se relacionen con diferentes actividades, no exclusivamente con la actividad comercial, ya que esto está sesgando las funciones del contador público a un solo tipo de organizaciones y se convierte en un inconveniente a la hora en que el profesional quiere asesorar en otro tipo de actividad.

Se recomienda a los programas de contaduría pública incluir en sus planes de estudio algunas bases de legislación de las actividades más representativas en la economía, principalmente bases sobre legislación minera, incluyendo sus características particulares como la elaboración de declaraciones de regalías; igualmente que se fortalezcan los temas de costos, salud ocupacional y análisis de la información financiera, ya que esto le permite al profesional abrirse a mayores oportunidades de trabajo.

\section{REFERENCIAS}

Argadoña, A. (1998, enero). La teoría de los stakeholders y el bien común. Documento de Investigación 355. Recuperado de http://www.iese.edu/research/pdfs/DI-0355.pdf

Carbal, A. (2009). Responsabilidad social empresarial y contabilidad: apuntes críticos. Recuperado de http://www.unilibrectg.edu.co/Descarga/PDF/Cien cias_Admon_y_Contables/RESPONSABILIDAD SOCEIAL.p $\overline{\mathrm{df}}$

Curvelo,, J. O. (2009). Ética y responsabilidad social del contador: perspectivas y tendencias frente a las IFRS. Recuperado de http://www.capic.cl/capic/images/vol7tema7.pdf

Esperanza, A. L.. (2010). La responsabilidad social y ambiental como componente fundamental en la formación del contador público udeceista. Recuperado de

http://190.27.248.91/redunicar/encuentros/5encuentro/pone ncias/010.pdf

Franco, R. (2002). Del hacer al saber, realidades y perspectivas de la educación contable en Colombia. 
Aunque los contadores encuestados (43\%) manifiestan dar a conocer en "gran parte" a los empresarios mineros la importancia de mantener adecuadas condiciones laborales y prácticas de seguridad, los empresarios expresan que en realidad lo que les recalcan sobre estos temas es poco (31\% de los empresarios manifiestan no recibir esta recomendación y un $10 \%$ medianamente).

Los contadores públicos informan que asesoran totalmente (30\% de los contadores encuestados) a los empresarios mineros sobre los derechos que por ley le corresponden a los trabajadores; mientras los empresarios en un $25 \%$ consideran que los están asesorando medianamente en cuanto al cumplimiento de sus obligaciones legales para con los trabajadores.

Los empresarios encuestados, en un $63 \%$, manifiestan que los contadores públicos que los asesoran no les dan a conocer en sus estados financieros aquellas situaciones que no les generan beneficio para la actividad.

Un $59 \%$ de los empresarios mineros encuestados considera que el contador público que los asesora no conoce bien la actividad minera que ellos desarrollan, ni sus grupos de interés. Afirmación ante la cual los contadores públicos encuestados indican, en un $30 \%$, que es verdad y otro $35 \%$ que sí los conocen bien.

Están totalmente de acuerdo tanto contadores $(61 \%$ de los encuestados) como los empresarios encuestados (59\%), en que no se han reunido para tratar situaciones o problemas relacionados con temas laborales, sociales y ambientales.

Por último, existe un acuerdo entre los diferentes grupos entrevistados en cuanto a que las condiciones de la minería mejorarían para todos gracias a un mayor asesoramiento y acompañamiento en el desarrollo de las actividades mineras por parte de los profesionales contadores, pues, de esta manera, se evitaría caer en el error de incumplir los compromisos y obligaciones que existen y todos los grupos de interés se verían beneficiados a corto, medio y largo plazo.

\section{CONSIDERACIONES FINALES}

El contador público, como profesional de una disciplina social, tiene un compromiso tanto social como profesional para con los grupos de interés de la organización que asesora, por eso él no puede limitar su labor a la asesoría en el cumplimiento de obligaciones legales, ni en el cumplimiento de una tarea limitada a rendir un informe netamente matemático, pues la responsabilidad social del profesional inicia desde el compromiso que tiene para con el empresario que lo contrata, sin importar cuál sea la actividad que este desarrolla, su compromiso está relacionado con el suministro de la información necesaria para el buen desarrollo de su actividad y con el apoyo constante ante la dudas y situaciones de dificultad que se puedan presentar en los diferentes ámbitos, económico, administrativo, social y ambiental.
El contador público, en su formación, adquiere las bases necesarias para prestar una asesoría integral en el desarrollo de su trabajo, sin embargo se encuentra que en los planes de estudio de formación se tiende a tratar principalmente temas relacionados con empresas comerciales y aspectos tributarios, lo que lleva a que el profesional se incline más por este tipo de organizaciones y funciones principalmente fiscales. Además, esto junto a la cultura económica global, conduce a cumplir con una tarea rutinaria y a no ofrecer más de la labor para la cual se fue contratado.

El contador público, al ofrecer una asesoría integral, involucrándose con los diferentes grupos de interés de las organizaciones en las que asesora, puede llegar a conocer y a comprender mejor la actividad que las empresas realizan y, en la medida en que sea posible, dar solución a situaciones de conflicto que se pueden presentar. Hay que aclarar que esta situación no lo lleva a hacer más de su trabajo sino, al contrario, a hacer mucho más enriquecedora su labor como asesor.

La responsabilidad social del contador está asociada al interés por la organización a la que presta sus servicios profesionales y sus grupos de interés, mediante el suministro de la información necesaria sobre los derechos y obligaciones que tiene la organización para con cada uno de ellos, para que de esta manera las organizaciones cumplan de una responsablemente con sus compromisos sociales y no caigan en error por desconocimiento u omisión. Por esto se considera importante que incorpore una serie de principios de responsabilidad social que lo lleven a desarrollar de una manera más adecuada su trabajo, generándose en él un compromiso social dentro de sus funciones laborales.

\section{CONCLUSIONES}

Existe un número importante de empresarios mineros que no utiliza los servicios de un contador. La mayoría de empresarios mineros manifiesta contratar sus servicios de manera ocasional a efectos de presentación de declaraciones y elaboración de balances, por lo tanto la participación de los contadores públicos en este sector es baja, y su mayor concentración se encuentra en las empresas comerciales.

Existe poca comunicación entre los contadores públicos y empresarios mineros, ya que la mayoría de ellos no realizan visitas a la organización que asesoran.

A pesar de que el profesional contable manifiesta conocer el concepto de responsabilidad social y considera que existe una responsabilidad social profesional y que su asesoría influye en los diferentes grupos de interés de las empresas que asesora, hay un desinterés por parte de él hacia las organizaciones mineras, así como hacia los empresarios y los demás grupos de interés de las mismas.
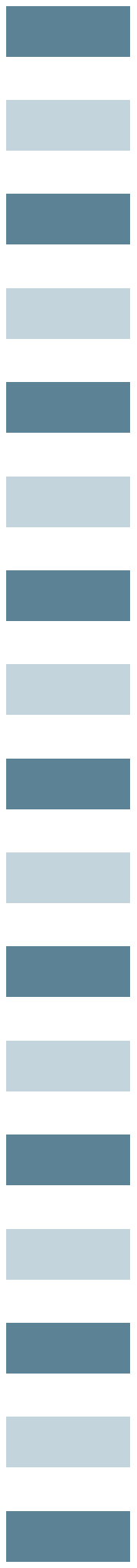
Machado, M. A. (2004). Revista de la Universidad de Antioquia, Contabilidad una Nueva Ciencia Social, (17-18), 272.

Machado, M.A. (2007) La responsabilidad social corporativa como resultante de un cambio en los imaginarios del contador público: del mecanicismo a la complejidad de las organizaciones. Contaduría Universidad de Antioquia. Recuperado de http://aprendeenlinea.udea.edu.co/revistas/index.p $\mathrm{hp} /$ cont/article/viewFile/2148/1750

Martínez, C. F. (2006). El deber ser de la contaduría pública en colombia. R ecuperado d e http://administracion.univalle.edu.co/Comunidad/ Memorias/evento1/archivos/E1\%20deber\%20ser $\% 20$ de $\% 201 \mathrm{a} \% 20$ contaduria $\% 20$ publica $\% 20$ en $\%$ 20Colombia.pdf

Ospina, E., Ruíz, A., Sotelo, M. (2011). La responsabilidad social: un valor agregado a la sociedad, al contador y a las organizaciones de hoy. $\mathrm{R}$ e c u p e r a d o d e http://aprendeenlinea.udea.edu.co/revistas/index.p hp/adversia/article/viewFile/10955/10048
Pérez, I. (2000). Dimensión ética de la responsabilidad social de la empresa con el medio ambiente. Recuperado de http://www.cicodi.org/Publicaciones/CDocuments andSettingscarmenEscritoriopublicacionesenviard imensioneticaempresamedioambiente21570552531.pdf

Pinzón, H. J. (2006). Responsabilidad del contador público. Recuperado de http://actualicese.com/actualidad/2006/12/04/resp onsabilidad-del-contador-publico/ parr.2,3,4.

Rivera, H. A. , Malaver,, M. N. (2011). La organización: los stakeholders y la responsabilidad social. Recuperado de http://www.urosario.edu.co/urosario_files/a0/a01 9d4bc-3670-4966-881c-ccaabf47d85 c.pdf

Salazar, C. M. (2002) El contador público y su compromiso social. Recuperado de . http://www.sappiens.com/CASTELLANO/articul os.nsf/Etica_y_Empresa/El_Contador_Público_y _su_compromiso_social/ 